\title{
Asistencia de los reclusos con problemas de salud mental en Holanda
}

\author{
G van 't Hoff, J de Looff, H Latooij
}

Senior Policy Adviser. National Agency of Correctional Institutions. Ministry of Justice

\section{RESUMEN}

En este trabajo, se ofrece una visión general de los presos que necesitan atención debido a un trastorno mental y que, por ese motivo han sido internados en lugares con atención especial, con distintos niveles de seguridad. Los presos con trastornos psíquicos son un factor importante en las instituciones penitenciarias en Holanda. Los servicios que existen actualmente para esos presos intentan ofrecer la mayor atención y apoyo posibles.

Palabras clave: Salud mental, psiquiatría forense, prisiones, Países Bajos.

\section{HEALTH CARE FOR PRISONERS WITH MENTAL HEALTH PROBLEMS IN HOLLAND}

\section{ABSTRACT}

In this study, a general picture is given of prisoners needing health care as a result of mental disorder and who, for this reason, have been interned in special care units with different security levels. Prisoners with mental disorders are an important factor in prisons in Holland. Services currently available for this type of inmate set out to offer the best care and support possible.

Key words: Mental health, forensic psychiatry, prisons Netherlands.

Texto recibido: Agosto 2008

Texto aceptado: Enero 2009

\section{INTRODUCCION}

Desde hace 30 años, ha aumentado enormemente en las instituciones penitenciarias el número de presos con un trastorno psíquico. En los Países Bajos, todos los años se encierra en torno a 70.000 presos. Una parte de ellos necesita atención debido a una afección psicológica o psiquiátrica. En este artículo, ofrecemos una visión general de las cantidades de presos que necesitan atención extra y que, por ese motivo han sido internados en lugares con atención especial, con distintos niveles de seguridad y de atención. Seguidamente, trataremos los establecimientos asistenciales de nueva formación y los cambios que han llevado a su creación.
Los servicios de salud mental para presos están sometidos a grandes cambios en estos momentos. Dirigido por la oferta y distribuido a pequeña escala por el país, este servicio deberá ofrecerse dentro de unos pocos años con una mayor orientación hacia la demanda y de forma concentrada en cinco ubicaciones. Para poder comprender en qué medida afecta todo esto al sistema actual de un servicio de salud mental de segunda línea para los presos prestado por especialistas, primero se comentará el aspecto que tiene ahora el sistema de atención y qué es lo que ha hecho que se ponga en movimiento todo el aparato. Después haremos un esbozo del aspecto que deberá tener idealmente el sistema de atención sanitaria dentro de cinco años. 


\section{NATURALEZA Y ALCANCE}

\author{
Enfermedades psiquiátricas de la población reclusa \\ en los Países Bajos
}

Antes de centrarnos en los diferentes departamentos sanitarios especializados dentro del aparato penitenciario holandés, vamos a mencionar las principales investigaciones realizadas desde los años ochenta sobre el alcance del grupo de presos que padecen trastornos psíquicos dentro del sistema penitenciario holandés, así como sobre su psicopatología.

- En 1980, la Comisión de Servicios Psiquiátricos/Terapéuticos del Sistema Penitenciario, del Ministerio de Justicia realizó una encuesta entre los directores de instituciones penitenciarias en los Países Bajos. Los resultados arrojan a una estimación del $10 \%$ de los presos con graves problemas psíquicos, cuyo comportamiento se relaciona con trastornos psíquicos.

- En 1989-1990, se realizó una investigación sobre la prevalencia de trastornos psíquicos entre presos con penas breves, de entre 18 y 24 años en el establecimiento penitenciario Nieuw Vossenveld (Bulten, 1998). Los presos fueron entrevistados poco después de su ingreso. Esta investigación arroja que el $79,9 \%$ de los presos, durante el año de la investigación o el año anterior a la investigación cumplen los criterios para un diagnóstico DIS-DSM-III eje-I o eje-II (los trastornos del eje-I incluyen adicciones a las drogas o al alcohol). El 32\% ha padecido un año antes o durante la investigación un trastorno del eje-I (excluidos los problemas de adicción), el $10 \%$ un trastorno afectivo, el $17 \%$ un trastorno de ansiedad y el $8 \%$ un trastorno de esquizofrenia/esquizofreniforme. Estas prevalencias son claramente superiores a las de la población general.

- Bleeker y Stalman (2007) informaron partiendo de datos del Servicio de Establecimientos Judiciales (Dienst Justitiële Inrichtingen, DJI)que la mitad de todos los presos padece un trastorno psiquiátrico (contando adicciones) según el DSM-IV-TR, que el 10\% necesita la ayuda de un psiquiatra y que el $4 \%$ de los presos no está en condiciones de estar en prisión como consecuencia del trastorno que padecen. Esperan que en realidad la prevalencia será mayor, porque los datos sólo se refieren a los trastornos que son conocidos por los psicólogos penitenciarios.
De los datos anteriores se desprende que los presos con trastornos psíquicos son un factor importante en las instituciones penitenciarias. Los servicios que existen actualmente para esos presos intentan ofrecer la mayor atención y apoyo posibles.

\section{PRINCIPIOS, LEY Y NORMATIVA}

En la Visión sobre la Atención Sanitaria del Servicio de Establecimientos Judiciales (2002), se constata que el respeto por los derechos fundamentales de los presos significa que en los establecimientos se ofrezca una atención sanitaria de la misma calidad que la que se ofrece en la sociedad libre. Los derechos y obligaciones de los presos se recogen en la Ley de Principios Penitenciarios (Penitentiaire Beginselenwet, $\mathrm{PBw}$ ) y en el Reglamento Penitenciario (Penitentiaire Maatregel, PM). La situación de prisión conlleva determinadas limitaciones, pero en ocasiones obliga a ofrecer una mayor atención. La legislación sanitaria holandesa (Ley sobre el contrato de tratamiento médico, Ley de profesiones en la atención sanitaria individual, Ley de Calidad de las entidades asistenciales) también se aplica en las instituciones penitenciarias. Los presos que tienen una queja sobre la sanidad pueden presentarla ante un asesor médico del Servicio de Establecimientos Judiciales o ante la Inspección de Sanidad del Ministerio de Sanidad, Bienestar y Deportes. Esta inspección comprueba la calidad de la sanidad y el cumplimiento de la legislación sanitaria por parte de todas las organizaciones médicas en los Países Bajos, también en las instituciones penitenciarias.

\section{ATENCIÓN DE $1^{a}$ LINEA: SERVICIO MÉDICO}

Los enfermeros judiciales son los primeros interlocutores de los presos que necesitan atención médica. En primera instancia, el enfermero o enfermera evalúa las quejas partiendo de tareas protocolizadas y delegadas, tras lo cual remitirá al preso al personal médico. Esto puede hacerse si el enfermero lo considera necesario, en caso de duda sobre la tramitación de la petición de ayuda o si lo pide expresamente el preso. El enfermero desempeña una función de puente entre el preso y el médico judicial, no desempeña el papel de filtro: el preso puede siempre consultar al médico judicial sin barreras. Los roles, derechos y obligaciones de los enfermeros y los médicos están descritos inequívocamente y su posición está claramente delimitada. 


\section{CONSULTAS MÉDICO-PSICOLÓGICAS}

En todos los establecimientos existen uno o varios psicólogos y psiquiatras forenses que forman parte del equipo del propio establecimiento y que pueden ser llamados a consulta por el servicio médico. La cooperación entre el servicio médico, el psicólogo del establecimiento y el psiquiatra forense adquiere forma en las consultas médico-psicológicas.

Las consultas médico-psicológicas (sigla en holandés: PMO que significa Psycho-medisch Overleg) son el órgano de consultas que se encarga de coordinar y asesorar sobre la asistencia en las instituciones penitenciarias. Se centra en las cuestiones individuales de asistencia y de ayuda para los presos con problemas psicosociales o psiquiátricos. Cualquiera de los participantes puede pedir que se comente el caso de un preso, pero también el propio preso o un funcionario (jefes de departamento y empleados del establecimiento penitenciario) pueden proponer la cuestión de un caso concreto. El director del establecimiento también puede solicitar asesoramiento sobre la supervisión de un preso individual o sobre la política o el ambiente existente en el establecimiento.

Las consultas médico-psicológicas están presididas por el psicólogo del establecimiento. El equipo se reúne como mínimo una vez por semana. Los miembros del órgano consultivo están obligados por el secreto profesional: los consejos al director u otros miembros de la organización sólo contienen la información necesaria para entender el comportamiento del preso y para supervisarlo de forma óptima. Los consejos se traducen en un plan de atención y en un plan de supervisión. Además, el órgano consultivo puede emitir asesoramiento sobre la forma de trabajo y el ambiente en el establecimiento y puede contribuir de esa forma a lograr un ambiente bueno y saludable en la prisión.

Las consultas médico-psicológicas tienen una importante tarea en la orientación de presos con afecciones psiquiátricas (graves) hacia departamentos especiales de supervisión, secciones internas de tratamiento o servicios externos de salud mental. Para conseguir un estándar de calidad, se ha desarrollado una "Mejor Práctica" (Best Practice ontwikkeling) de las consultas médico-psicológicas. La intención es que, en su día, todas las consultas médico-psicológicas se organicen de la misma forma. Al hacer más transparentes los procesos de trabajo, es posible realizar controles de calidad y, si es necesario, orientar el proceso. Una vez aplicadas, estas mejores prácticas significarán una enorme profesionalización de la atención en las prisiones.

\section{SERVICIOS ESPECIALES}

\author{
Secciones con un nivel especial de atención \\ y seguridad
}

En este momento, el servicio de salud mental en el sistema penitenciario está dividido en 26 pequeñas secciones, en unas diez ubicaciones esparcidas por todo el país. Las secciones surgieron debido a una necesidad de atención específica y de control para los presos con problemas psiquiátricos, que no pueden controlarse dentro del régimen regular. Muchas instituciones penitenciarias disponen de una sección de atención especial donde puede internarse a presos con problemas mentales o especialmente vulnerables, para una especial supervisión y observación. Si esto no es suficiente, el preso puede ser trasladado a un establecimiento nacional para supervisión intensiva, acogida en crisis o tratamiento. Naturalmente, la seguridad de estos establecimientos se adapta a su población. También hay posibilidades para presos con problemas de conducta, que necesitan medidas extra de seguridad. Estas secciones de control están divididas en secciones para, entre otros, personas con limitaciones de adaptarse a la sociedad. También existe una sección nacional de aislamiento para presos que deben cumplir una sanción disciplinaria. Se trata de un total de 500 celdas destinadas a estas formas especiales de atención que se combinan parcialmente con el control.

\section{Observación forense y secciones de tratamiento}

Cuando un preso sufre una crisis psiquiátrica grave en algún lugar de los Países Bajos, el psiquiatra que trabaja en el establecimiento penitenciario en cuestión lo comunica a la sección de observación y tratamiento forense (siglas holandesas: FOBA) Es posible, por ejemplo, un internamiento urgente para evitar un peligro grave. Anualmente, se realizan en esa sección unos 325 ingresos.

Todas las unidades en la FOBA se caracterizan por haber sido creadas en pequeña escala. Existe espacio para la supervisión individual y el programa diurno se ofrece según el personal disponible. El objetivo es ayudar a los presos lo mejor posible a pasar su periodo en prisión, sin que sufran "daños penitenciarios”. También se intenta que se les traslade lo antes posible a los establecimientos públicos de los servicios de salud mental, para que empiecen ya el tratamiento durante su periodo de prisión y, de esta forma, puedan continuar el tratamiento ya iniciado en los 
servicios públicos de salud mental después de salir del marco judicial. No obstante, el traslado suele enfrentarse con dificultades. Las causas son distintas: una de ellas son las diferencias culturales entre el Ministerio de Justicia y el Ministerio de Sanidad, Bienestar y Deportes. Mientras el Ministerio de Justicia tiene como tarea primaria garantizar la seguridad y, de esta forma, ofrecer asistencia en un marco forzoso, la sanidad pública se centra en el problema de asistencia (voluntaria) del paciente. No es ninguna sorpresa que ambas posturas choquen.

\section{Dimensiones de las secciones de atención especial}

Para la descripción de la psicopatología de los presos en las distintas secciones de atención especial, nos limitamos a las secciones de atención especial y a las secciones (nacionales) de supervisión individual. El inventario del Servicio de Establecimientos Judiciales de los grupos especiales en el sistema penitenciario holandés en 2007 proporciona información sobre la psicopatología de los presos en estas secciones. Los resultados más curiosos son que el 37\% de los presos en una sección de atención especial padece esquizofrenia u otra psicosis y que el $45 \%$ tiene un trastorno de personalidad del grupo $\mathrm{B}$. Al parecer, en las secciones (nacionales) de supervisión individual, entre el $80 \%$ y el $95 \%$ de los presos padece una enfermedad psiquiátrica. Entre el $40 \%$ y el $70 \%$ de los casos, se trata de esquizofrenia u otra enfermedad psicótica. La co-morbididad con la adicción y un trastorno de personalidad es alta. La prevalencia de las distintas enfermedades en estas secciones es relativamente constante desde hace años.

\section{CAMBIO}

\section{De asistencia penitenciaria a curativa}

Mientras durante las últimas décadas ha aumentado paulatinamente el número de presos con un trastorno psiquiátrico en el sistema penitenciario, cada vez está más claro que es de una importancia esencial una buena transición entre la asistencia penitenciaria y la asistencia curativa.

Tres cambios que surgen prácticamente de forma paralela, se ocupan de que se renueve el sistema de servicios de salud mental para los presos.

El primer cambio se produce dentro del sistema penitenciario bajo el nombre Modernización del Sis- tema Penitenciario (Modernisering Gevangeniswezen, MGW). Este programa es la continuación del anterior proyecto Prisión y Tratamiento a Medida para Adultos (Detentie en Behandeling op Maat voor volwassenen, DBM-v). El punto de partida del DBMv y del MGW para los servicios de salud mental para los presos es que se realice un trabajo a medida y de forma eficaz. La modernización del sistema penitenciario parte de la estandarización y uniformidad siempre que sea posible y del trabajo a medida cuando sea necesario. Para poder ofrecer ese trabajo a medida de forma eficaz, la atención clínica forense dentro del sistema penitenciario deberá concentrarse en cinco ubicaciones.

Asimismo, desde los años ochenta existe un aumento de presos con trastornos psiquiátricos. El sistema penitenciario y los establecimientos psiquiátrico-forenses apenas no podían mantenerse al ritmo de los cambios, con lo cual eran incapaces de proporcionar suficiente asistencia. Pero los servicios de salud mental en los Países Bajos tampoco estaban suficientemente equipados para tratar a este grupo de pacientes difíciles. Varios incidentes graves con expresos fueron motivo de una enorme intranquilidad social y de la correspondiente atención política. Esto se tradujo en 2006 en la institución de una comisión investigadora parlamentaria (en lo sucesivo: la Comisión Visser). Una de las recomendaciones de la Comisión Visser se refería a la investigación de las posibilidades de un hospital psiquiátrico penitenciario, debido a la creciente problemática psiquiátrica en el sistema penitenciario. No obstante, la problemática puede ser de tal naturaleza que no siempre es directamente visible, esto es, la llamada psiquiatría oculta. Asimismo, la Comisión Visser constató una escasez de plazas apropiadas para el ingreso de un número de estos presos. En el plan de enfoque, la recomendación de la Comisión Visser es el internamiento en un centro psiquiátrico, lo que debe traducirse en una ampliación sustancial del número de plazas de atención psiquiátrica para los presos.

Finalmente, una moción del miembro del Senado holandés Van de Beeten en la que se pide al gobierno que se ocupe de la necesaria coherencia entre los servicios curativos y penitenciarios, llevó al asesoramiento de la Comisión interministerial Houtman sobre la gestión y la financiación de la asistencia en un marco judicial, centrado en una mejor conexión de la asistencia penitenciaria en la asistencia curativa. En este asesoramiento, la Comisión Houtman propuso traspasar el presupuesto para la asistencia forense del presupuesto del Ministerio de Sanidad, Bienestar y Deportes al presupuesto del Ministerio de Justicia. De 
esta forma, Justicia puede adquirir su propia asistencia en el marco judicial, y garantizar mejor la responsabilidad de la ejecución de las sanciones y medidas penales. El asesoramiento de la Comisión Houtman ha sido suscrito en el informe de la Comisión Visser y la ejecución del mismo ha sido incluida en el programa Renovación de la Asistencia Forense (Vernieuwing Forensische Zorg, VFZ). Los puntos de partida y las partes de este programa también se aplican a la asistencia psiquiátrica para presos prestada por especialistas (de segunda línea).

\section{¿Qué significa esto concretamente para los servicios de salud mental para presos prestados por especialistas (de segunda línea) en el sistema penitenciario?}

Desde el programa Modernización del Sistema Penitenciario (MGW), primero se concentrarán las secciones existentes de pequeña escala en cinco ubicaciones, que se repartirán por el país. De esta forma, podrán emplearse de forma más eficaz y eficiente los recursos para la asistencia y se espera que el aumento de la escala conlleve un mejoramiento de la calidad. Para seleccionar los presos adecuados que puedan necesitar asistencia, se desarrolla un instrumento de screening. El objetivo es que todos los presos que entran en el sistema penitenciario regular sean controlados para ver si tienen alguna indicación de asistencia forense, con el fin de poder empezar temprano a ofrecer asistencia a medida.

La recomendación del parlamento de ampliar el número de plazas de asistencia para los presos llevará seguidamente a un aumento de 700 plazas además de las 500 ya existentes. Se trata de una ampliación cualitativa; las plazas regulares ya existentes serán transformadas en plazas asistenciales. El nivel de asistencia de las plazas que se van a crear en el sistema penitenciario será comparable al de los servicios públicos de salud mental, entendiendo que deben tenerse en cuenta las limitaciones que conlleva la situación de detención.

En el sistema penitenciario se van a introducir también tres elementos del programa de Renovación de la Asistencia Forense. El punto de partida "comprar a menos que...” se aplicará a las plazas extra de servicios de salud mental para presos que van a crearse. De las 700 plazas de ampliación, 350 plazas se comprarán a los servicios públicos de salud mental a través de un procedimiento de adjudicación.

Para mejorar la conexión con el sector sanitario público, también en lo relativo a la financiación, se ha optado por utilizar el mismo sistema de financiación que en los servicios públicos de salud mental para los cinco establecimientos asistenciales. Se utilizarán las combinaciones de diagnóstico y tratamiento (siglas en holandés: DBC) que han sido complementadas con un componente específico de Justicia: la seguridad (esto es: combinaciones de diagnóstico y tratamiento con seguridad) (DBBC). El sistema de indicación no sólo dice algo sobre la necesidad de asistencia, sino también sobre el nivel de seguridad necesario para evitar reincidencia. El sistema de indicación se ajusta en su terminología lo más posible al idioma de las combinaciones de diagnóstico y tratamiento con la seguridad.

En la transición de la prisión a los servicios públicos de salud mental, el sistema debe mostrar lo que ha ofrecido como tratamiento hasta ese momento dentro de la prisión. La asistencia pública puede continuar "sencillamente" donde terminó la Justicia.

Finalmente, un procedimiento uniforme de indicación debe procurar que todos los presos acaben en la cama correcta.

\section{¿Cómo se ajustan finalmente todos estos elementos?}

Los próximos años, se llevarán a cabo todos los cambios comentados. En primer lugar, se ha dado comienzo a la ampliación de la oferta de asistencia en las cinco ubicaciones. Las preparaciones para la concentración también están en marcha. En 2009 y 2010 deberán tener ya forma. Esto significa concretamente que en varias ubicaciones desaparecerán las secciones en pequeña escala para volver a instalarse en una ubicación asistencial. Una ubicación asistencial tendrá como mínimo 120 plazas asistenciales y como máximo unas 250 plazas. Este cambio en las dimensiones exige otra visión de la organización de la oferta de asistencia distinta de la actual. La actual organización de la oferta asistencial en pequeñas secciones, cada una con sus características específicas, hace que la oferta y la demanda no siempre estén coordinadas. La consecuencia son largas listas de espera periódicas y, a veces, plazas vacías porque no hay presos suficientes que cumplan el perfil de la sección. Para evitar estos problemas en los cinco nuevos establecimientos asistenciales, se ha desarrollado una nueva visión de la asistencia en colaboración con las cinco ubicaciones. Esa visión de la asistencia parte de un máximo de tres niveles de asistencia de intensidad creciente. Asimismo, cada nivel asistencial tiene un nivel de control que puede intensificarse según la composición de la población reclusa en el momento concreto. Este siste- 
ma hace que los establecimientos asistenciales sean máximamente flexibles para dar forma y organizar la oferta asistencial. De esta forma, puede aprovecharse al máximo la capacidad de las celdas.

Tal y como ya se ha dicho, es importante ocuparse de que los presos acaben en el lugar correcto dentro de este nuevo sistema asistencial. Para empezar, se está creando un nuevo instrumento de screening. Al entrar en un establecimiento penitenciario, cada preso será controlado según un procedimiento fijo para comprobar si tiene una posible necesidad de asistencia. En primera instancia, se controlará si existe uno o más de los siguientes cinco terrenos problemáticos:

- Trastornos psicóticos.

- Trastornos de ansiedad y del ánimo.

- Deficiencia mental.

- Adicción.

- Trastorno invalidante, trastorno de la personalidad.

Si unos diez días después de su ingreso resulta que puede existir un trastorno, el caso del preso en cuestión será comentado en las consultas médico-psicológicas del establecimiento penitenciario. Si este órgano consultivo opina que la asistencia que puede ofrecer el establecimiento no es suficiente, se emitirá una indicación con ayuda del instrumento de indicación especialmente desarrollado al efecto. Este instrumento se utilizará para indicar todos los servicios de salud mental de segunda línea en un marco penal, incluida la asistencia para personas con deficiencia mental y la asistencia para adictos.

El punto de partida es que la indicación sea emitida por un agente indicador independiente. Si se sospecha que es necesaria atención clínica, será un psiquiatra del Instituto Holandés de Psiquiatría y Psicología Forenses. Una indicación de asistencia ambulatoria podrá ser emitida tanto por un psicólogo (independiente) como por el funcionario de reinserción social.

Si se emite una indicación para asistencia clínica, se plantea la cuestión de si puede efectuarse un ingreso en los servicios públicos de salud mental o si va a realizarse el ingreso en uno de los cinco establecimientos asistenciales. Aquí tiene un voto importante el ministerio público. El internamiento en los servicios públicos de salud mental sólo puede efectuarse si el ministerio público está conforme. Esto tiene que ver con la responsabilidad del ministerio público por la ejecución de las sanciones penales. Si existe acuerdo, se efectuará el traslado a una institución. Si el ministerio público considera demasiado arriesgado el ingreso en los servicios públicos de salud mental o si se trata de un delito que ha levantado una enorme intranquilidad social, entonces seguirá el ingreso en un establecimiento asistencial del sistema penitenciario. También se efectuará el ingreso en un establecimiento asistencial penitenciario si no puede facilitarse la combinación de asistencia y seguridad.

La oferta de asistencia en las cinco ubicaciones está centrada, no sólo en un empleo flexible del aspecto controlador, sino también en los cinco aspectos problemáticos en que se centra el screening. Se están desarrollando y, en su momento, se ofrecerán programas asistenciales para cada uno de los problemas o para una combinación de los mismos.

¿Qué conlleva toda la operación?

1. se pretende una mejor conexión entre asistencia penitenciaria y curativa

2. se pretende que el paciente apropiado acabe en la cama apropiada

3. se pretende un cambio cualitativo con respecto a la asistencia que proporciona el sistema penitenciario

4. se pretende contribuir a reducir la reincidencia

¡Dentro de unos años sabremos si hemos tenido éxito!

\section{CORRESPONDENCIA}

Talleres Editoriales Cometa, S.A.

Ctra. Castellón, km. 3,400.

50013 Zaragoza.

\section{REFERENCIAS BILBIOGRÁFICAS}

1. Bleeker J.A.C., en Stalman P.F.A (2007). De psychiatrie in het Nederlandse recht. (La psiquiatría en el derecho holandés) Capítulo 2007; 10: 161-173.

2. Bleeker, J.A.C., Stalman, P.F.A. (2007). Psychiatrische voorzieningen in het gevangeniswezen. Van bescherming en begeleiding naar behandeling. (Servicios psiquiátricos en el sistema penitenciario. De protección y supervisión a tratamiento). En: De psychiatrie in het Nederlandse recht. (La psiquiatría en el derecho holandés). Bajo redacción de Raes, B.C.M., Bakker, F.A.M., 2007: p. 161-173.

3. Bulten. B.H. (1998). Gevangen tussen straf en zorg. Psychische stoornissen bij jeugdige, kortgestrafte gedetineerde. 1998 (Presos entre la pena y la 
asistencia. Trastornos psíquicos de los presos jóvenes y con penas cortas).

4. Fazel\&Danesh (2002). Serious mental disorder in 23.000 prisoners: a systematic review of 62 surveys (Trastornos mentales graves en 23.000 presos: revisión sistemática de 62 investigaciones), Lancet 2002, 545-550.

5. Huygen e.a. (2007). Begeleidingsvisie FOBA, 20073-7.

6. Ministerio de Justicia (1983). Informe de la Comisión de Servicios Psiquiátricos/Terapéuticos del Sistema Penitenciario. (Commissie Psychiatrische/Therapeutische Voorzieningen Gevangeniswezen) 1983.

7. Ministerio de Justicia (1994). Informe del grupo parcial del proyecto 'Psychisch gestoorde gedetineerden' (Presos con trastornos psíquicos) en el marco del proyecto Werkzame Detentie (Prisión Provechosa) 1994.

8. Ministerio de Justicia (2001). Zorg om Zorg. Een inventarisatie van de knelpunten rond de zorg voor psychisch gestoorde gedetineerden. 2001 (Prestar atención a la asistencia. Un inventario de los puntos conflictivos en torno a la asistencia a los presos con trastornos psíquicos). Servicio de Instituciones Judiciales, Dirección del Sector del Sistema penitenciario.

9. Ministerio de Justicia (2004). Toegeleiding. Instrumentenset Penitentiair Verpleegkundige, 2004 (Explicación. Instrumentario del Enfermero Penitenciario). Servicio de Instituciones Judiciales.
10. Ministerio de Justicia (2006). Jaarverslag monitoring 2006 (B) IBA 2005 (Informe anual de supervisión (B) IBA 2005). Servicio de Instituciones Judiciales.

11. Ministerio de Justicia (2008). Bijzondere Groepen II Gevangeniswezen (Grupos Especiales II Sistema Penitenciario). Een inventarisatie van de populatie, het zorg-en het beveiligingsaanbod van de BZA, VBA, ISD, JOVO en de zedenafdeling in het Gevangeniswezen. 2008 (Un inventario de la población, la oferta de asistencia y de seguridad de BZA, VBA, ISD, JOVO y la sección de delitos sexuales en el Sistema Penitenciario). Servicio de Instituciones Judiciales.

12. Schoemaker, C., Zessen, G. van (1997). Psychische stoornissen bij gedetineerden. Een verkennend onderzoek in Penitentiair Complex Scheveningen. 1997 (Trastornos psíquicos de los presos. Una investigación reconocedora en el Complejo Penitenciario de Scheveningen).

13. Smit, N.W. de. (1989). Psychose en Gevangenis. (Psicosis y Prisión). En: Justitiële Verkenningen (Reconocimientos Judiciales), año 15, número 2 (Diferentie in detentie (Diferencia en prisión)), 1989: p. 62-76.

14. WHO Health in prison project (2008). The Trencin statement on prisons and mental health 2008 (OMS Proyecto de salud en la prisión (2008). La declaración de Trencin sobre prisiones y salud mental). 